\title{
Evodiamine sensitizes U87 glioblastoma cells to TRAIL via the death receptor pathway
}

\author{
MUHAMMAD KHAN $^{1,3}$, YANYING BI ${ }^{2}$, JAVED IQBAL QAZI ${ }^{3}$, LIMEI FAN ${ }^{4}$ and HONGWEN GAO ${ }^{1}$ \\ ${ }^{1}$ Department of Pathology, The Second Hospital of Jilin University, Changchun 130041; ${ }^{2}$ Infection Control Office, \\ The First Affilliated Hospital to Changchun University of Chinese Medicine, Changchun 130021, P.R. China; \\ ${ }^{3}$ Department of Zoology, University of the Punjab, Quaid-e-Azam Campus, Lahore 54590, Pakistan; \\ ${ }^{4}$ Department of Gynecology and Obstetrics, The Second Hospital of Jilin University, Changchun 130041, P.R. China
}

Received November 6, 2013; Accepted May 2, 2014

DOI: $10.3892 / \mathrm{mmr} .2014 .2705$

\begin{abstract}
The tumor necrosis factor- $\alpha$-related apoptosis-inducing ligand (TRAIL) has been shown to selectively induce death in cancer cells without affecting healthy cells. Most glioma cells are resistant to TRAIL-induced apoptosis. Resistance to TRAIL limits its potential use as a drug for therapy of glioma. The present study was conducted to identify bioactive compounds that have the potential to sensitize U87 glioblastoma cells to TRAIL. Evodiamine, a major bioactive compound of the Chinese herb Evodiae fructus, has been reported to sensitize U87 glioblastoma cells to TRAIL. TRAIL and evodiamine, in combination or alone, were used to treat U87 glioblastoma cells. We show that evodiamine treatment inhibited cell growth in a dose-dependent manner; however, TRAIL alone failed to exert any cytotoxic effect. Combining TRAIL with evodiamine significantly increased the apoptotic rate of U87 glioblastoma cells, as compared to evodiamine treatment alone. Further investigation of the mechanism underlying these effects revealed that the evodiamine + TRAIL effect is associated with the increased expression of death receptor (DR)4, DR5, caspase-8 and cleaved caspase-3. The present study demonstrated, for the first time to the best of our knowledge, that evodiamine can sensitize U87 glioblastoma cells to TRAIL via the death receptor pathway. Thus, our results suggest that combined treatment with evodiamine and TRAIL may represent a novel chemotherapeutic strategy for the therapy of glioma.
\end{abstract}

Correspondence to: Dr Hongwen Gao, Department of Pathology, The Second Hospital of Jilin University, 218 Ziqiang Street, Changchun 130041, P.R. China

E-mail: gaohongwen@jlu.edu.cn

Dr Limei Fan, Department of Gynecology and Obstetrics, The Second Hospital of Jilin University, 218 Ziqiang Street, Changchun 130041, P.R. China

E-mail: fanlimei1971@126.com

Key words: evodiamine, TRAIL, U87 cells, DR4, caspase-8, caspase-3

\section{Introduction}

Glioblastoma multiform (GBM) is the most malignant, aggressive and devastating primary brain tumor, accounting for $\sim 80 \%$ of malignant gliomas (1-4). Despite concerted efforts to improve the currently available therapies, the prognosis of glioblastoma remains very poor, with a mean patient survival of 12 months following diagnosis $(1,2,5,6)$. At present, treatment of malignant gliomas is one of the most difficult challenges in oncology (7). Exploring novel chemotherapeutic strategies is therefore necessary for improving the outcome of glioblastoma treatment strategies.

The tumor necrosis factor- $\alpha$-related apoptosis-inducing ligand (TRAIL), also called Apo2L, has been reported to selectively induce apoptosis in various cancer cells, by binding to the death receptors TRAIL-R1/DR4 and TRAIL-R2/DR5 (8). TRAIL binds to DR4/DR5, to subsequently interact with the adaptor protein known as FAS-associated death domain protein (FADD) and procaspase-8, and form the death-inducing signaling complex (DISC). Within this complex, FADD recruits procaspase- 8 , which becomes activated. Activated caspase- 8 then directly activates the downstream effector caspase-3, resulting in cell death via the type I extrinsic apoptotic pathway, or cleaves Bid, a pro-apoptotic member of the Bcl-2 family, leading activation of the type II extrinsic apoptotic pathway (9-11). TRAIL is considered to be the safest and the most promising cell death-inducing ligand for clinical application, due to its selective cytotoxicity towards cancer cells $(7,12)$. Previous studies have shown that most glioma cells are more or less resistant to the apoptotic effect of TRAIL $(7,9,10,13,14)$. However, an increasing body of literature has provided evidence that glioma cells can be sensitized to TRAIL-induced apoptosis with different chemotherapeutic agents. Combined treatment with drugs that can sensitize glioma cells to TRAIL may ultimately increase its potency $(9-11,13)$. Thus, identification of novel chemotherapeutic agents that are capable of sensitizing glioma cells to TRAIL is highly desirable.

Evodiamine is one of the major components of the Evodiae fructus. An increasing number of reports in the past decade have documented its broad-spectrum cytotoxicity towards various cancer cell lines of different origin, through 
difstinct mechanisms (15-25). In the present study, we report that evodiamine not only inhibits cell growth, but also sensitizes U87 glioblastoma cells to TRAIL-induced apoptosis, by inducing the expression of death receptors.

\section{Materials and methods}

Reagents and antibodies. All the chemicals were purchased from Sigma-Aldrich (Beijing, China) unless otherwise stated. Evodiamine was purchased from Tauto Biotech. Co., Ltd. (Shanghai, China) and purity ( $>99 \%$ ) was determined by HPLC, conducted at the Central Research Lab of Bethune Second Hospital of Jilin University (Changchun, China) as described in previous studies $(15,19)$. The chemical structure of evodiamine is shown in Fig. 1A. The apoptosis assay kit was purchased from Nanjing KeyGen Biotech Co., Ltd. (Nanjing, China). Antibodies specific to caspase-8, cleaved caspase-3 and $\beta$-actin were purchased from Beyotime Institute of Technology (Shanghai, China), whereas the antibody targeting death receptor (DR) 5 was purchased from Cell Signaling Technology (Danvers, MA, USA). Horseradish peroxidaseconjugated secondary antibodies (goat anti-rabbit and goat anti-mouse) were purchased from Sigma-Aldrich. The enzyme-linked immunosorbent assay (ELISA) kit for human DR4 was purchased from ROTRN Shanghai Biotechnology Co., Ltd. (Shanghai, China). TRAIL was purchased from Abcam Inc. (Cambridge, MA, USA).

Cell cultures and treatment. U87 glioblastoma cells were obtained from the American Type Culture Collection (ATCC; Manassas, VA, USA) and were maintained in Dulbecco's modified Eagle's medium supplemented with $10 \%$ fetal bovine serum in $5 \% \mathrm{CO}_{2}$ at $37^{\circ} \mathrm{C}$. Cells were treated with evodiamine dissolved in dimethyl sulfoxide (DMSO), at a final DMSO concentration of $0.5 \%$, or with DMSO alone for $24 \mathrm{~h}$. DMSO-treated cells were used as the control.

Determination of cell viability by the MTT assay. Cell viability was determined by the MTT [3-(4,5-dimethylthiazol2-yl)-2,5-diphenyltetrazolium bromide] assay as previously described (26). Briefly, U87 glioblastoma cells were treated with various concentrations of evodiamine $(0-20 \mu \mathrm{M})$ or TRAIL $(0-50 \mathrm{ng} / \mathrm{ml})$ for $24 \mathrm{~h}$. Following treatment, the MTT reagent was added $(500 \mu \mathrm{g} / \mathrm{ml})$ and cells were further incubated at $37^{\circ} \mathrm{C}$ for $4 \mathrm{~h}$. Subsequently, $150 \mu \mathrm{l}$ of DMSO were added to dissolve the formazan crystals, and the absorbance was measured at $570 \mathrm{~nm}\left(\mathrm{~A}_{570}\right)$ in a Multiskan ${ }^{\mathrm{TM}}$ GO Microplate spectrophotometer (Thermo Fisher Scientific Inc., Waltham, MA, USA). The percentage of cell viability was calculated as follows: Cell viability $(\%)=\left(\mathrm{A}_{570}\right.$ sample $-\mathrm{A}_{570}$ blank $)$ ( $\mathrm{A}_{570}$ control - $\mathrm{A}_{570}$ blank) x 100.

Live/Dead assay. Living and dead cells were quantified using the fluorescent probes calcein acetoxymethylester (AM) and propidium iodide (PI) as previously described $(1,27)$. Calcein AM is highly lipophilic and cell membrane-permeable. In viable cells, it is converted into calcein by esterases and emits strong green fluorescence. Thus, it stains only viable cells. PI, a dye used for nuclear staining, is cell membrane-impermeable. Cells with impaired plasma membrane integrity are stained red due to the entry of PI and failure to retain calcein. Since both calcein and PI are excited at $490 \mathrm{~nm}$, simultaneous monitoring of viable and dead cells is feasible under a fluorescence microscope (1X71; Olympus Corporation, Tokyo, Japan). To quantify living and dead cells, the cells were treated with evodiamine $(10 \mu \mathrm{M})$ and TRAIL $(50 \mathrm{ng} / \mathrm{ml})$ either separately or in combination (evodiamine $10 \mu \mathrm{M}+$ TRAIL $50 \mathrm{ng} / \mathrm{ml}$ ) for $24 \mathrm{~h}$. Following incubation, cells were collected, washed with phosphate-buffered saline (PBS), and incubated with PBS containing $2 \mu \mathrm{M}$ calcein AM and $4 \mu \mathrm{M} \mathrm{PI}$, for $20 \mathrm{~min}$ in the dark, at room temperature. A total of 100 cells were then counted under the fluorescence microscope in order to quantify the viable and dead cells.

Analysis of apoptosis by flow cytometry. U87 glioblastoma cells were treated with evodiamine and TRAIL either separately or in combination, for $24 \mathrm{~h}$, as described in the Live/Dead assay. Following treatment, cells were harvested, washed with PBS and resuspended in $500 \mu \mathrm{l}$ of binding buffer, containing $5 \mu \mathrm{l}$ Annexin V-fluorescein isothiocyanate (FITC) and $5 \mu 1$ PI. The cells were left in the dark for 15 min according to the kit instructions (KeyGen). The percentage of apoptotic cells was evaluated on a Epics XL flow cytometer (Beckman Coulter, Brea, CA, USA).

Assessment of DR4 expression by enzyme-linked immunosorbent assay (ELISA). The level of DR4 in U87 glioblastoma cells was measured spectrophotometrically using the human DR4 ELISA kit. Briefly, U87 cells were treated with $10 \mu \mathrm{M}$ evodiamine for $24 \mathrm{~h}$. Following treatment, cells were collected by centrifugation $(700 \mathrm{x} \mathrm{g})$, and cell lysates were prepared according to the kit instructions. The level of DR4 in the cell lysates was quantified spectrophotometrically at $450 \mathrm{~nm}$ on a Synergy H1 Hybrid Microplate Reader (H1MD; Bio-Tek, Beijing, China).

Immunoblotting. U87 glioblastoma cells were treated with evodiamine $(10 \mu \mathrm{M})$ and TRAIL $(50 \mathrm{ng} / \mathrm{ml})$ either separately or in combination (evodiamine $10 \mu \mathrm{M}+$ TRAIL $50 \mathrm{ng} /$ $\mathrm{ml}$ ), for $24 \mathrm{~h}$. Following treatment, cells were collected by centrifugation, and proteins were isolated as previously described $(27,28)$. The protein concentrations were determined on a NanoDrop 1000 (Thermo Fisher Scientific Inc.) spectrophotometer. A total of $30 \mu \mathrm{g}$ of protein was subjected to $12 \%$ sodium dodecyl sulfate polyacrylamide gel electrophoresis and transferred onto a polyvinylidine difluoride membrane. After blocking with 5\% (w/v) non-fat milk and washing with Tris-buffered saline with Tween-20 (TBST), the membrane was incubated for $2 \mathrm{~h}$ at room temperature with antibodies targeting DR5 (dilution, 1:1,000), cleaved caspase-3 $(1: 1,000)$, caspase- $8(1: 1,000)$ and $\beta$-actin $(1: 400)$. After washing, the blot was incubated with horseradish peroxidase-conjugated goat anti-rabbit or -mouse IgG secondary antibodies $(1: 5,000)$ for $1 \mathrm{~h}$ at room temperature. Following a TBST wash, the signals were detected using a ChemiLucen ${ }^{\mathrm{TM}}$ Plus kit (EMD Millipore, Billerica, MA, USA).

Statistical analysis. Results were expressed as mean \pm standard error of the mean (SEM). Comparisons between two groups were performed with Student's t-tests, and between 
A<smiles>CN1c2ccccc2C(=O)N2CCc3c([nH]c4ccccc34)C21</smiles>

B

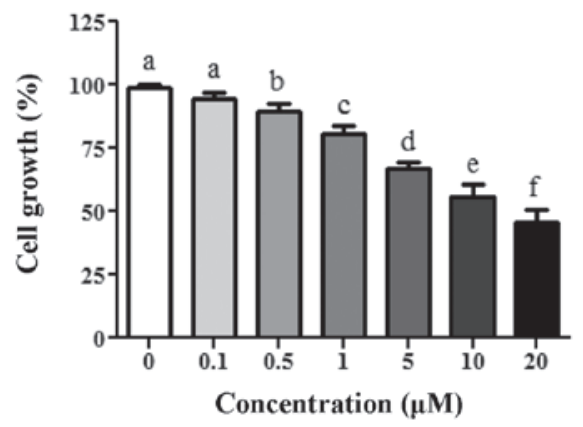

C

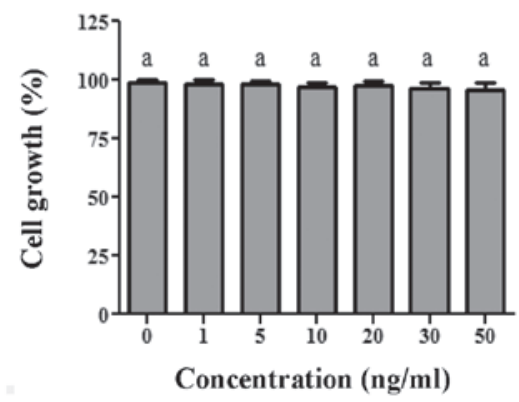

Figure 1. MTT cell viability assay after treatment with evodiamine and tumor necrosis factor- $\alpha$-related apoptosis-inducing ligand (TRAIL). (A) Chemical structure of evodiamine. (B and C) U87 glioblastoma cells were treated with indicated concentrations of (B) evodiamine and (C) TRAIL for 24 h, and cell viability was determined by the MTT assay. Bars denote standard error of the mean (SEM) from three independent experiments. Columns not sharing the same letter denote significantly different data $(\mathrm{P}<0.05)$.

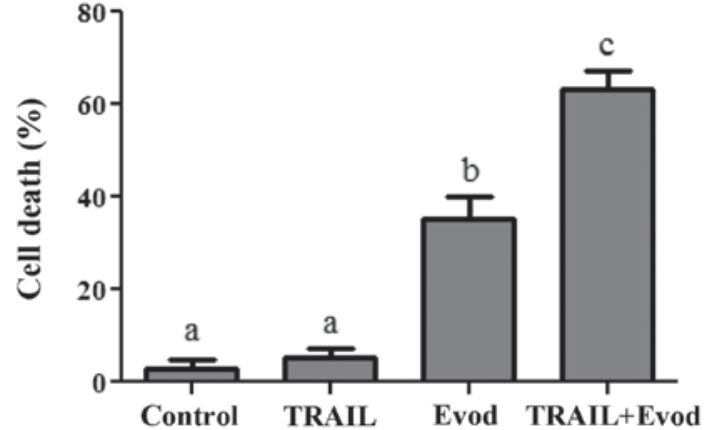

Figure 2. Evodiamine sensitizes U87 glioblastoma cells to tumor necrosis factor- $\alpha$-related apoptosis-inducing ligand (TRAIL). U87 glioblastoma cells were treated with TRAIL $(50 \mathrm{ng} / \mathrm{ml})$, evodiamine (Evod; $10 \mu \mathrm{M})$ or a combination of both for $24 \mathrm{~h}$, and cell death was examined using the Live/Dead assay. Bars denote standard error of the mean (SEM) from three independent experiments. Columns not sharing the same letter denote significantly different data $(\mathrm{P}<0.05)$. Control, cells treated with dimethyl sulfoxide.

multiple groups with a one-way analysis of variance (ANOVA), followed by the Tukey's Multiple Comparison test. $\mathrm{P}<0.05$ was considered to indicate statistically significant differences.

\section{Results}

Evodiamine sensitizes U87 glioblastoma cells to TRAIL. First, we measured the inhibitory effect of evodiamine and TRAIL on growth of U87 glioblastoma cells using the MTT assay. Evodiamine significantly inhibited the growth of U87 cells in a dose-dependent manner, as shown in Fig. 1B. The half maximal inhibitory concentration $\left(\mathrm{IC}_{50}\right)$ value of evodiamine against U87 glioblastoma cells was $12 \mu \mathrm{M}$. However, TRAIL (1-50 ng/ml) alone did not inhibit the growth of U87 glioblastoma cells within $24 \mathrm{~h}$. These results were further confirmed by the Live/Dead assay, as shown in Fig. 2.
Next, we investigated whether evodiamine can sensitize U87 glioblastoma cells to TRAIL. For this purpose, we treated the cells with $10 \mu \mathrm{M}$ evodiamine and $50 \mathrm{ng} / \mathrm{ml}$ TRAIL for $24 \mathrm{~h}$. The combined treatment significantly increased cell death in U87 glioblastoma cells as compared to evodiamine treatment alone (Fig. 2). The above-mentioned concentrations of evodiamine and TRAIL were employed in all cotreatment experiments performed in this study.

Evodiamine + TRAIL induce apoptotic cell death in U87 glioblastoma cells. To confirm that evodiamine + TRAIL-induced cell death occurs via apoptosis, we performed flow cytometry analysis. Cells were treated with evodiamine $(10 \mu \mathrm{M})$ and TRAIL (50 ng/ml) either separately or in combination for $24 \mathrm{~h}$, and the percentages of cells undergoing apoptosis/necrosis were determined after staining with Annexin V-FITC and PI. As expected, TRAIL alone did not induce apoptosis, while evodiamine significantly induced apoptosis in U87 glioblastoma cells after $24 \mathrm{~h}$ (Fig. 3). Combined treatment with evodiamine and TRAIL significantly enhanced apoptosis compared to treatment with each drug alone. The data demonstrated that evodiamine sensitizes U87 glioblastoma cells to TRAIL-induced apoptosis.

Evodiamine induces DR4 and DR5 expression. Since U87 glioblastoma cells are resistant to TRAIL-mediated apoptosis, a number of chemotherapeutic agents have been investigated for their ability to sensitize tumor cells to TRAIL-mediated apoptosis by inducing the expression of death receptors. Therefore, we examined here whether evodiamine can induce the expression of DR4 and DR5 in U87 glioblastoma cells. We measured the expression of DR4 and DR5 in U87 glioblastoma cells before and after treatment with evodiamine, using ELISA and western blot analysis, respectively. As shown in Fig. 4, evodiamine treatment increased the expression of both DR4 and DR5 as compared to the control. 
A

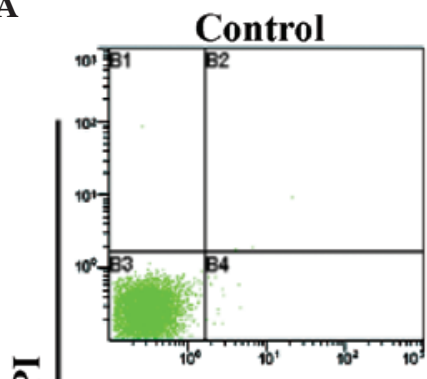

$\bar{a}$

Evod

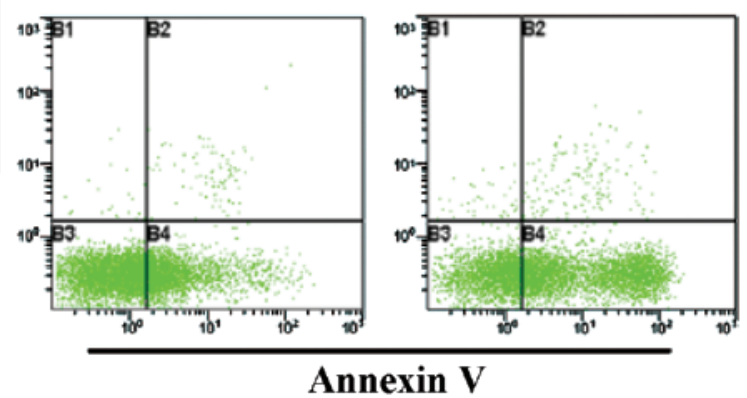

B

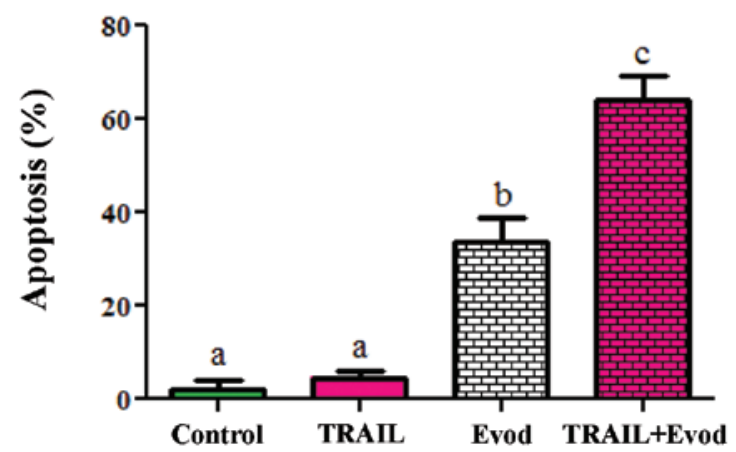

Figure 3. Flow cytometry analysis of apoptosis. (A) U87 glioblastoma cells were treated with tumor necrosis factor- $\alpha$-related apoptosis-inducing ligand (TRAIL; $50 \mathrm{ng} / \mathrm{ml}$ ), evodiamine (Evod; $10 \mu \mathrm{M}$ ) or a combination of both for $24 \mathrm{~h}$, and apoptosis was determined by flow cytometry following staining with Annexin V-fluorescein isothiocyanate (FITC)/propidium iodide (PI). (B) Bars denote standard error of the mean (SEM) from three independent experiments. Columns not sharing the same letter denote significantly different data $(\mathrm{P}<0.05)$. Control, dimethyl sulfoxide-treated cells.

Evodiamine treatment increases TRAIL-induced apoptosis through caspase activation. To further evaluate the activation of downstream signaling related to apoptosis following the increased expression of DR4 and DR5, we measured the expression of caspase-8 and caspase-3. As shown in Fig. 5, TRAIL treatment alone did not affect the level of caspase- 8 and cleaved (activated) caspase-3; however, evodiamine induced the expression of caspase- 8 and cleaved caspase-3. Combined treatment with evodiamine and TRAIL further increased the expression of caspase- 8 and cleaved caspase- 3 . These data suggested that the apoptotic effect of evodiamine + TRAIL may be due to caspase activation.

\section{Discussion}

Drug resistance is one of the major obstacles to the successful treatment of cancer (29). An ideal anticancer chemotherapeutic

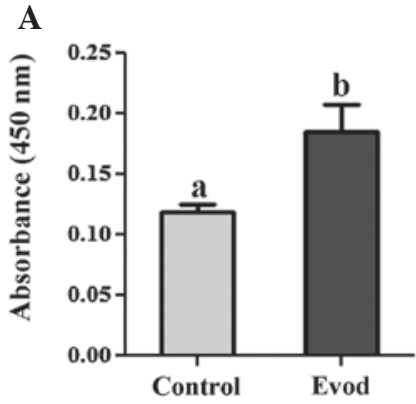

B

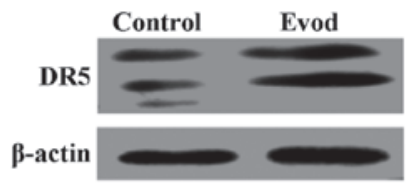

Figure 4. Evodiamine induces the expression of human death receptor (DR)4 and DR5. (A) U87 glioblastoma cells were treated with evodiamine (Evod; $10 \mu \mathrm{M}$ ) for $24 \mathrm{~h}$. The level of DR4 was then measured spectrophotometrically using an enzyme-linked immunosorbent assay (ELISA) kit. Bars denote standard error of the mean (SEM) from three independent experiments. Columns not sharing the same letter denote significantly different data $(\mathrm{P}<0.05)$. (B) Total cell lysates were extracted from control (dimethyl sulfoxide-treated) and evodiamine-treated cells. A total of $30 \mu \mathrm{g}$ of protein was subjected to western blotting in order to detect DR5, using $\beta$-actin as the loading control.

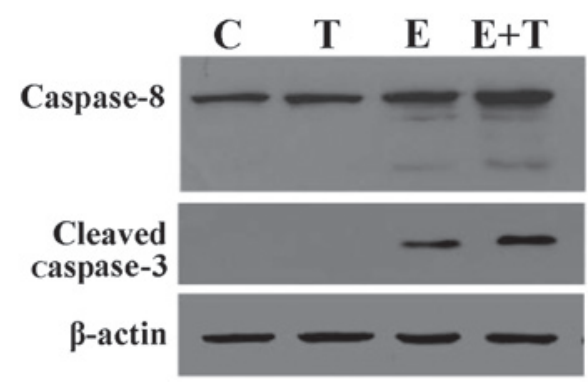

Figure 5. Activation of caspases induced by evodiamine (E) and tumor necrosis factor- $\alpha$-related apoptosis-inducing ligand (TRAIL; T). U87 glioblastoma cells were treated with TRAIL $(50 \mathrm{ng} / \mathrm{ml})$, evodiamine $(10 \mu \mathrm{M})$ or a combination of both for $24 \mathrm{~h}$. The expression of caspase- 8 and cleaved caspase- 3 was determined by western blot analysis, using $\beta$-actin as the loading control. C, control (dimethyl sulfoxide-treated) cells.

agent does not only kill cancer cells, but further exhibits a high degree of selective toxicity towards these cells compared to healthy ones (2). A few reports previously indicated that TRAIL selectively induces apoptosis in cancer cells with no or minimal toxicity on healthy cells $(30,31)$. However, most glioma cells, including U87 glioblastoma cells, are resistant to TRAIL-induced apoptosis (9-11). Therefore, developing new treatment strategies to overcome resistance is important for the successful application of TRAIL in clinical therapy. The present study aimed to identify a therapeutic agent that, in addition to sensitizing glioma cells to TRAIL, exhibits promising antiglioma activity.

Evodiamine, a major bioactive constituent of the Evodiae fructus has a broad-spectrum of cytotoxicity in various cancer cell lines, including glioma cell lines (15-25). Therefore, we tested evodiamine's potential to sensitize U87 glioblastoma cells to TRAIL-mediated apoptosis. First, we examined the antiglioma activity of evodiamine and TRAIL using the MTT assay. We found that evodiamine inhibits the growth of U87 glioblastoma cells in a dose-dependent manner, while TRAIL failed to inhibit growth of U87 glioblastoma cells. The results are in agreement with a previous report on evodiamine-induced cell death in U87 glioblastoma cells (25), and with other 
reports on resistance of this cell line to TRAIL-induced apoptosis $(9,11,14)$. Next, we applied a combinatorial treatment with evodiamine and TRAIL on U87 glioblastoma cells. We found that combined application of evodiamine and TRAIL significantly reduces the viability of U87 glioblastoma cells, and induces apoptotic cell death, as shown in Figs. 2 and 3. We therefore demonstrated, for the first time to the best of our knowledge, that evodiamine is a potent sensitizer of U87 glioblastoma cells to TRAIL.

Resistance to TRAIL-induced apoptosis can occur at different levels of the apoptotic cascade, through downregulation of death receptors, induction of inhibitors of apoptosis, and defects in FADD and caspase-8 (11). A large body of evidence suggests that several therapeutic agents can increase the sensitivity of glioma cells to TRAIL-induced apoptosis $(9,11,14,31,32)$. TRAIL-resistant cancer cell lines can be sensitized to TRAIL by inducing the expression of TRAIL receptors (TRAIL-R1 and TRAIL-R2) using different therapeutic drugs $(7,14)$. The data of the present study indicated that combined treatment with evodiamine and TRAIL significantly increases apoptotic cell death in U87 glioblastoma cells compared to either drug alone. In order to ascertain the mechanism by which evodiamine increases TRAIL-induced apoptosis in U87 glioblastoma cells, we measured the expression of death receptors (DR4 and DR5). We found that evodiamine treatment markedly increases the expression of both DR4 and DR5 in U87 glioblastoma cells.

Once TRAIL binds to death receptors, it recruits the cytoplasmic adaptor protein FADD. The recruited FADD interacts with procaspase- 8 or procaspase- 10 through its death effector domain to form DISC. DISC promotes the activation of caspase-8, which in turn induces apoptotic cell death by activating the effector caspase, i.e. caspase-3, either directly or indirectly via the mitochondrial apoptotic pathway $(9-11,31)$. To investigate whether the increased apoptotic cell death in U87 glioblastoma cells observed upon combined treatment with evodiamine and TRAIL is due to activation of caspase- 8 , we measured the expression of caspase- 8 in U87 glioblastoma cells treated with evodiamine and TRAIL or each drug separately. In line with the results from the apoptosis assay, the expression of caspase- 8 was higher in cells treated with evodiamine and TRAIL as compared to the cells treated with evodiamine alone. This finding provided preliminary evidence that increased apoptotic cell death in U87 glioblastoma cells may be due to the activation of caspase- 8 by evodiamine and TRAIL. This result is further supported by a previous study, which reported that evodiamine induces the activation of caspase-8 in hepatocellular carcinoma cells (18). Next, we measured the expression of cleaved caspase-3 in U87 glioblastoma cells. The results provided additional support for the activation of the apoptotic pathway by the evodiamine and TRAIL combination, since the activated form of caspase- 3 was increased in cells treated with evodiamine + TRAIL. To unequivocally confirm these results, a future study will systematically assess the levels of both the inactive and cleaved form of caspase in evodiamine + TRAIL-treated cells.

In conclusion, our data provide novel evidence that evodiamine efficiently sensitizes U87 glioblastoma cells to TRAIL-induced apoptosis by inducing the expression of DR4 and DR5. Thus, combined treatment with evodiamine and TRAIL may represent a useful strategy to counter the resistance of cancer cells to apoptosis, and provides new opportunities for glioblastoma treatment. Additional studies are however needed to investigate the exact molecular mechanism by which evodiamine sensitizes U87 glioblastoma cells to TRAIL.

\section{Acknowledgements}

This study was supported by grants from the National Natural Science Foundation of China (no. 81201268) and the National Natural Science Foundation of Jilin Province (no. 201215057).

\section{References}

1. Khan M, Yi F, Rasul A, Li T, Wang N, Gao H, Gao R and Ma T: Alantolactone induces apoptosis in glioblastoma cells via GSH depletion, ROS generation, and mitochondrial dysfunction. IUBMB Life 64: 783-794, 2012.

2. Khan M, Zheng B, Yi F, Rasul A, Gu Z, Li T, Gao H, Qazi JI, Yang $\mathrm{H}$ and Ma T: Pseudolaric Acid B induces caspase-dependent and caspase-independent apoptosis in U87 glioblastoma cells. Evid Based Complement Alternat Med 2012: 957568, 2012.

3. Amantini C, Mosca M, Nabissi M, Lucciarini R, Caprodossi S Arcella A, Giangaspero F and Santoni G: Capsaicin-induced apoptosis of glioma cells is mediated by TRPV1 vanilloid receptor and requires p38 MAPK activation. J Neurochem 102: 977-990, 2007.

4. Su Y, Li G, Zhang X, Gu J, Zhang C, Tian Z and Zhang J: JSI-124 inhibits glioblastoma multiforme cell proliferation through $\mathrm{G}(2) / \mathrm{M}$ cell cycle arrest and apoptosis augment. Cancer Biol Ther 7: 1243-1249, 2008.

5. Yin D, Wakimoto N, Xing H, Lu D, Huynh T, Wang X, Black KL and Koeffler HP: Cucurbitacin B markedly inhibits growth and rapidly affects the cytoskeleton in glioblastoma multiforme. Int J Cancer 123: 1364-1375, 2008.

6. Jeon JY, An JH, Kim SU, Park HG and Lee MA: Migration of human neural stem cells toward an intracranial glioma. Exp Mol Med 40: 84-91, 2008.

7. Qiu B, Sun X, Zhang D, Wang Y, Tao J and Ou S: TRAIL and paclitaxel synergize to kill U87 cells and U87-derived stem-like cells in vitro. Int J Mol Sci 13: 9142-9156, 2012.

8. Suliman A, Lam A, Datta R and Srivastava RK: Intracellular mechanisms of TRAIL: apoptosis through mitochondrial-dependent and -independent pathways. Oncogene 20: 2122-2133, 2001.

9. Siegelin MD, Reuss DE, Habel A, Rami A and von Deimling A: Quercetin promotes degradation of survivin and thereby enhances death-receptor-mediated apoptosis in glioma cells. Neuro Oncol 11: 122-131, 2009.

10. Siegelin MD, Reuss DE, Habel A, Herold-Mende C and von Deimling A: The flavonoid kaempferol sensitizes human glioma cells to TRAIL-mediated apoptosis by proteasomal degradation of survivin. Mol Cancer Ther 7: 3566-3574, 2008.

11. Xia S, Li Y, Rosen EM and Laterra J: Ribotoxic stress sensitizes glioblastoma cells to death receptor induced apoptosis: requirements for c-Jun $\mathrm{NH}_{2}$-terminal kinase and Bim. Mol Cancer Res 5: 783-792, 2007.

12. Smyth MJ, Takeda K, Hayakawa Y, Peschon JJ, van den Brink MR and Yagita H: Nature's TRAIL - on a path to cancer immunotherapy. Immunity 18: 1-6, 2003.

13. Knight MJ, Riffkin CD, Muscat AM, Ashley DM and Hawkins CJ: Analysis of FasL and TRAIL induced apoptosis pathways in glioma cells. Oncogene 20: 5789-5798, 2001.

14. Badr CE, Wurdinger T, Nilsson J, Niers JM, Whalen M, Degterev A and Tannous BA: Lanatoside C sensitizes glioblastoma cells to tumor necrosis factor-related apoptosis-inducing ligand and induces an alternative cell death pathway. Neuro Oncol 13: 1213-1224, 2011.

15. Khan M, Qazi JI, Rasul A, Zheng Y and Ma T: Evodiamine induces apoptosis in pancreatic carcinoma PANC-1 cells via NF $\kappa$ B inhibition. Bangl J Pharmacol 8: 8-14, 2013.

16. Wang KL, Hsia SM, Yeh JY, Cheng SC, Wang PS and Wang SW: Anti-proliferative effects of evodiamine on human breast cancer cells. PloS One 8: e67297, 2013. 
17. Tu YJ, Fan X, Yang X, Zhang $\mathrm{C}$ and Liang HP: Evodiamine activates autophagy as a cytoprotective response in murine Lewis lung carcinoma cells. Oncol Rep 29: 481-490, 2013.

18. Yang J, Cai X, Lu W, Hu C, Xu X, Yu Q and Cao P: Evodiamine inhibits STAT3 signaling by inducing phosphatase shatterproof 1 in hepatocellular carcinoma cells. Cancer Lett 328: 243-251, 2013.

19. Rasul A, Yu B, Zhong L, Khan M, Yang H and Ma T: Cytotoxic effect of evodiamine in SGC-7901 human gastric adenocarcinoma cells via simultaneous induction of apoptosis and autophagy. Oncol Rep 27: 1481-1487, 2012.

20. Zhang C, Fan X, Xu X, Yang X, Wang X and Liang HP: Evodiamine induces caspase-dependent apoptosis and $\mathrm{S}$ phase arrest in human colon lovo cells. Anticancer Drugs 21: 766-776, 2010.

21. Wang C, Li S and Wang MW: Evodiamine-induced human melanoma A375-S2 cell death was mediated by PI3K/Akt/caspase and Fas-L/NF-kappaB signaling pathways and augmented by ubiquitin-proteasome inhibition. Toxicol In Vitro 24: 898-904, 2010.

22. Yang J, Wu LJ, Tashino S, Onodera S and Ikejima T: Reactive oxygen species and nitric oxide regulate mitochondria-dependent apoptosis and autophagy in evodiamine-treated human cervix carcinoma HeLa cells. Free Radic Res 42: 492-504, 2008.

23. Kan SF, Yu CH, Pu HF, Hsu JM, Chen MJ and Wang PS: Anti-proliferative effects of evodiamine on human prostate cancer cell lines DU145 and PC3. J Cell Biochem 101: 44-56, 2007.

24. Lee TJ, Kim EJ, Kim S, Jung EM, Park JW, Jeong SH, Park SE, Yoo YH and Kwon TK: Caspase-dependent and caspase-independent apoptosis induced by evodiamine in human leukemic U937 cells. Mol Cancer Ther 5: 2398-2407, 2006.
25. Liu AJ, Wang SH, Chen KC, Kuei HP, Shih YL, Hou SY, Chiu WT, Hsiao SH and Shih CM: Evodiamine, a plant alkaloid, induces calcium/JNK-mediated autophagy and calcium/mitochondria-mediated apoptosis in human glioblastoma cells. Chem Biol Interact 205: 20-28, 2013.

26. Khan M, Ding C, Rasul A, Yi F, Li T, Gao H, Gao R, Zhong L, Zhang K, Fang $\mathrm{X}$ and Ma T: Isoalantolactone induces reactive oxygen species mediated apoptosis in pancreatic carcinoma PANC-1 cells. Int J Biol Sci 8: 533-547, 2012.

27. Khan M, Yu B, Rasul A, Al Shawi A, Yi F, Yang H and Ma T: Jaceosidin induces apoptosis in U87 glioblastoma cells through G2/M phase arrest. Evid Based Complement Alternat Med 2012: 703034, 2012

28 Khan M, Li T, Ahmad Khan MK, Rasul A, Nawaz F, Zheng Y and Ma T: Alantolactone induces apoptosis in HepG2 cells through GSH depletion, inhibition of STAT3 activation, and mitochondrial dysfunction. Biomed Res Int 2013: 719858, 2013.

29. Melguizo C, Prados J, Luque R, Ortiz R, Rama AR, Caba O, Rodriguez-Serrano F, Alvarez PJ and Aranega A: Modulation of multidrug resistance gene expression in peripheral blood mononuclear cells of lung cancer patients and evaluation of their clinical significance. Cancer Chemother Pharmacol 71: 537-541, 2013.

30. Voelkel-Johnson C: An antibody against DR4 (TRAIL-R1) in combination with doxorubicin selectively kills malignant but not normal prostate cells. Cancer Biol Ther 2: 283-290, 2003.

31. Reis CR, van der Sloot AM, Natoni A, Szegezdi E, Setroikromo R, Meijer M, Sjollema K, Stricher F, Cool RH, Samali A, Serrano L and Quax WJ: Rapid and efficient cancer cell killing mediated by high-affinity death receptor homotrimerizing TRAIL variants. Cell Death Dis 1: e83, 2010.

32. Keane MM, Ettenberg SA, Nau MM, Russell EK and Lipkowitz S: Chemotherapy augments TRAIL-induced apoptosis in breast cell lines. Cancer Res 59: 734-741, 1999. 